\title{
Enseñar a aprender con metodología activa en el Grado en Bellas Artes
}

\author{
María del Carmen BeLlido MÁrqueZ \\ Universidad de Granada \\ cbellido@ugr.es
}

Recibido: $21 / 11 / 2012$

Aceptado: 23/01/2013

\begin{abstract}
Resumen
Ante el planteamiento de la adaptación y desarrollo del Plan de Bolonia al Grado en Bellas Artes, surge la necesitad de nuevas metodologías educativas que modifiquen los roles tradicionales del profesorado y del alumnado según las nuevas premisas del Espacio Europeo de Educación Superior. Desde la asignatura Escultura III: Metodologías escultóricas (Grupo D), se realiza una propuesta metodológica llevada a cabo durante el curso académico 2011-2012 y se analizan sus resultados. Dicha actividad promueve la mejora del proceso de enseñanza-aprendizaje, mediante metodologías activas que se basan en la resolución de problemas y en el trabajo colaborativo.
\end{abstract}

Palabras clave: Docencia universitaria, metodologías activas e innovación docente.

\section{Teaching to Learning with Active Methodology in the Fine Arts Degree}

\begin{abstract}
Given the approach of the adaptation and development of the Bologna Plan Degree in Fine Arts, it comes up the need for new educational methodologies that changes the traditional roles of teacher and students to new premises of the European Higher Education Area. We have made a methodology carried out during the academic year 2011-2012 in the subjet Sculpture III: Scuptural Methods (Group D), and to have analyzed their results. This activity increases improving teaching-learning process, by proposing active methodologies problem solving and collaborative work.
\end{abstract}

Keywords: University teaching, active methodologies and teaching innovation.

\section{Referencia normalizada}

BELLIDO MÁRQUEZ, María del Carmen (2013): "Enseñar a aprender con metodología activa en el Grado en Bellas Artes". Estudios sobre el mensaje periodístico. Vol. 19, Núm. especial abril, págs.: 593603. Madrid, Servicio de Publicaciones de la Universidad Complutense.

Sumario: 1. Introducción. 2. Metodología. 3 Desarrollo; 3.1 Tareas del profesorado y del alumnado; 3.2 Instrumentos de evaluación. 4. Resultados. 5. Conclusiones. 6. Referencias bibliográficas.

\section{Introducción}

El Plan de Bolonia plantea una actividad docente derivada de la distribución de objetivos, contenidos y competencias que el alumno debe adquirir en cada materia para superar las asignaturas que componen las diferentes titulaciones universitarias.

En este cambio profundo de las enseñanzas superiores surgen dificultades que requieren nuevos replanteamientos de la didáctica universitaria que actualicen las metodologías, realicen planificaciones previas y utilicen instrumentos de evaluación eficaces (García-Valcárcel, 2001:73). El interés y flexibilidad del docente en este proceso de adaptación a los nuevos retos es de vital importancia para ir obteniendo resultados positivos gradualmente (Zabalza, 2007: 97-107), (Cedeño, 2009: 3-5). De igual forma, los recursos formativos y herramientas docentes que las universidades ofrecen para conseguir estos logros son de la misma importancia. Por su parte, el alumno deber adaptarse a nuevas metodologías de trabajo necesarias en el adecuado 
proceso de su aprendizaje (Zabalza, 2007: 179-190). Por lo tanto, la adaptación de las nuevas metodologías docentes es una tarea conjunta entre la institución académica, el profesorado y el alumnado (Contreras et al., 2005: 15-18).

El Grado en Bellas Artes de la Universidad de Granada se está implantado progresivamente desde el curso académico 2009-2010 tal como consta en el Documento Verifica del Grado en Bellas Artes (Universidad de Granada, 2010: web); esto está requiriendo nuevas formas de enseñanza-aprendizaje. Para ello, se apuesta por metodologías docentes activas (Pagés et. al. 2010:165-175), apoyadas en la formación del profesorado (Zabalza, 2007: 143-178) y en el desarrollo de Proyectos de Innovación Docente (Benito y Cruz, 2005: 21-50), (Ubieto et al. 2008: 25).

Las actividades de enseñanza-aprendizaje mediante metodologías aplicadas al planteamiento de proyectos y resolución de problemas están siendo iniciativas que se adaptan bien a las prácticas docentes del Grado en BB. AA., dado que las Guías de muchas de sus asignaturas cuenta con una importante carga de enseñanza práctica, que requieren planteamientos de proyectos creativos (dibujo, pintura, escultura, nuevas tecnologías, fotografía, grabado, diseño, etc.) (Pagés et al., 2010: 155-164).

Este artículo presenta el diseño, puesta en práctica y resultados de estrategias docentes activas aplicadas a la asignatura Escultura III: Metodologías escultóricas (Grupo D), desarrolladas durante el curso académico 2011-2012, contemplando ciertos contenidos y competencias específicas que figuran en su Guía Docente (Universidad de Granda, 2012: web). Los resultados académicos reflejan la adquisición de competencias del alumnado al concluir la actividad que ha contado con una evaluación cuantitativa y cualitativa.

El objetivo general de la iniciativa es mejorar la calidad educativa de la asignatura que sirve de marco en el presente proyecto (Escultura: Metodologías escultóricas, Grupo D), mediante la actualización de métodos de enseñanza-aprendizaje, basados en metodologías activas del saber ser/ saber estar, acordes con los requerimientos del Espacio Europeo de Enseñanza Superior (Universidad Rey Juan Carlos, 2008: web) que potencien la educación colaborativa en beneficio del aprendizaje del alumno (Cross et al., 2007: 17,31), a través de la resolución de problemas propuestos llevados a proyectos creativos.

Para conseguir el amplio objetivo general es necesario definir otros más específicos que lo diversifiquen:

1. Poner a disposición del alumnado metodologías de trabajo que le ayude a desarrollar hábitos académicos en los que la investigación sea una metodología activa del aprendizaje, que repercutirá positivamente en su formación, gracias a la adquisición de competencias transversales (Zabalza et al. 2010: 178) que le dotarán de capacidades útiles en su formación universitaria e inclusión en el mundo laboral.

3. Mejorar el rendimiento académico de los estudiantes en el área docente abordada (la escultura) de forma específica y en la titulación del Grado en Bellas Artes en general, mediante la participación del alumno en las clases, su planificación de las tareas a realizar y el aprovechamiento de su trabajo autónomo, en un ambiente docente agradable que potencie el crecimiento personal de los implicados (profesorado y alumnado) (García y Manzano, 2010: 110). 
4. Desarrollar estrategias de aprendizaje y formación del alumnado, como el trabajo colaborativo (Hernández y Olmos 2011: web), la búsqueda y selección de información, la responsabilidad en el trabajo individual y compartido, la exposición en público, la auto-evaluación y la co-evaluación.

\section{Metodología}

La elección de la metodología conlleva algunos planteamientos previos a considerar relacionados con los objetivos a conseguir, donde se contempla: mejorar el aprendizaje de los estudiantes (Villar, 2004: 265-321), promover una nueva visión del trabajo del profesor como docente y vehículo para el aprendizaje (Gros y Romaná, 2004: 147-156), mejorar los rendimientos de la titulación académica con el compromiso y apoyo de la institución universitaria y aproximarse, cada vez más, a los métodos docentes subyacentes en los nuevos planteamientos de la construcción del EEES (Rué, 2007: 21-55).

Partiendo de estos principios, se elige una metodología activa adecuada según la materia a impartir y la innovación docente a realizar, teniendo presente las posibilidades de éxito, las ventajas y los inconvenientes previstos, a solventar (Pagés et al., 2010: 165-173). En definitiva, se busca una coherencia metodológica acorde con los objetivos propuestos, la disciplina a impartir y los medios disponibles, tanto humanos como materiales (López, 2005: 8). Todo ello atendiendo a las competencias específicas que figuran en la Guía de la Asignatura (Universidad de Granada, 2012: web). Entre éstas se eligen las siguientes:

» "1. Conocimiento del vocabulario, códigos y conceptos inherentes a la creación escultórica.

»2. Conocimiento de los materiales y de los procesos derivados de la creación y/ o producción escultórica.

»3. Conocimiento de los métodos y técnicas de producción asociados a los lenguajes de carácter tridimensional."

Se seleccionan dos temas de la Guía Docente (Universidad de Granada, 2012: web) que abordan contenidos que permiten ser desarrollados en la actividad. Estos son:

»"Tema 1. Profundización en el vocabulario y lenguaje plástico en la práctica escultórica.

» Tema 3. Exposición-reflexión panorámica e histórica sobre la evolución en el uso y significado de materiales, técnicas y procedimientos escultóricos".

Se diseña una metodología basada en la realización de proyectos, mediante la resolución de problemas. Los alumnos deben desarrollar sus proyectos creativos atendiendo a los problemas contemplados en el enunciado del ejercicio a realizar (Mazarío, 2009: web).

En esta actividad el docente deber ser un agente conductor que oriente y estimule los progresos del estudiante hasta la adquisición de resultados positivos (Cedeño, 2009: 15). Para ello se plantea la realización de trabajos colaborativos (Cross et al., 2007: 17-31) hechos por grupos reducidos, pues estos propician el compromiso cola- 
borativo, agudizan la responsabilidad del alumno y son un modelo acertado para generar conocimiento grupal y múltiple (Exley y Dennick, 2009: 9-21).

La propuesta se realiza en varias sesiones de clases presenciales y en las correspondientes horas de trabajo autónomo del alumno. Los integrantes de cada grupo deben asistir a un mínimo de tres clases para la realización del trabajo: elección y diseño del proyecto (1), desarrollo del mismo (2) y preparación para su exposición (3); además se comprometen a asistir a las exposiciones y defensas de los proyectos de los otros grupos.

La actividad comprende acciones cuantitativas y cualitativas, según la perspectiva de la titulación (artísticas, técnicas, etc.) que mejoran la realidad formativa de los alumnos. La evaluación del trabajo forma parte de la evaluación final, y se refleja en las calificaciones definitivas.

El profesorado planifica, presenta y controla el desarrollo de la actividad propuesta en forma de Innovación Docente (Zabalza y Zabalza, 2010: 85-94), informando a los alumnado de cómo se hará su valoración y cómo afectará en la calificación final. Presenta toda la información en la Guía Docente de la Asignatura. Él es un guía del desarrollo del trabajo (Gros y Romaná, 2004: 147-156) que facilita las técnicas de búsqueda de información, supervisa la organización de los grupos y atiende las consultas de los alumnos en las tutorías y en las clases teóricas y prácticas (Gómez 2011: 111-119).

Los componentes de los grupos de trabajo serán elegidos por los alumnos. Estos han de estar formados por cinco o seis miembros y contar con un portavoz. Además, el docente nombra a dos estudiantes de la clase como coordinadores de toda la actividad.

Las tutorías adquieren relevancia en las consultas del alumnado y la orientación del profesor, quien de esta manera puede atender a personas o grupos de forma específica. Así, las actividades tutoriales se cargan de contenido y utilidad apropiados (Exley y Dennick, 2009).

Se potencia el trabajo colaborativo grupal, además del aprendizaje propio del alumno, quien debe responsabilizarse de su parte del ejercicio y del buen funcionamiento del grupo (Cross et al. 2007: 17-31). Se desarrolla la auto-evaluación formativa y la crítica constructiva. Se califica la exposición, el diseño del proyecto, las actividades de campo, la auto-evaluación individual y la grupal o co-evaluación.

La actividad es evaluada por alumnado y profesorado. El primero hace una evaluación de su propio trabajo personal, del ejercicio en grupo y de la experiencia docente (metodología utilizada). El segundo evalúa la superación de objetivos propuestos, la experiencia docente (enseñanza-aprendizaje), el rendimiento del estudiante, la calidad de la docencia y la actividad de forma global.

El trabajo cuenta con un cronograma de planificación temporal para las acciones a realizar por profesorado y alumnado, desarrollándose dentro de la programación de la asignatura. Se efectúa en tres semanas lectivas que suponen doce horas de clase presencial y sus correspondientes de trabajo autónomo del alumno. La primera semana se dedica a la presentación del ejercicio, elección de grupo, definición de propuestas, planteamientos iniciales y adquisición de información documental; la segunda 
al desarrollo y realización del trabajo y la tercera a la redacción, presentación y defensa pública del mismo.

El enunciado del ejercicio es el siguiente: realizar y exponer un proyecto escultórico en grupos de cinco a seis personas. Dicho proyecto tendrá presente su ubicación en el exterior (material perdurable), lugar de ubicación (zona pública de una ciudad), tema (representativo de esa población y su ubicación), y un presupuesto limitado por la entidad que encargara el proyecto, de ser un caso real. El ejercicio debe contener una parte escrita (texto explicativo), parte visual (imágenes, planos, bocetos bidimensionales y tridimensionales) y una maqueta a escala de la propuesta escultórica definitiva a realizar. Los trabajos se presentan en clase con el apoyo visual de un PowerPoint.

El alumnado dispone en la Facultad de Bellas Artes de Granada con los fondos documentales de la biblioteca, los espacios-talleres de escultura y su infraestructura para la realización del ejercicio. Todas las actividades docentes de la asignatura tienen el apoyo metodológico de los medios TICs ofrecidos por la Universidad de Granada, como la Plataforma Swad, el Tablón de Docencia y el correo institucional de profesores y alumnos. Estos medios favorecen el aprendizaje colaborativo mediante el uso nuevas tecnologías (Hernández y Olmos, 2011: web).

\section{Desarrollo}

\subsection{Tareas del profesorado y del alumnado}

El profesorado define, concreta y diseña la actividad y su adaptación a cada una de las competencias a tratar. Previamente se documenta sobre casos de estudio precedentes para hacer una propuesta coherente y factible (Herreid, 1994: 221-229), (Bernes, 1994: web).

Tareas del profesorado:

» Presentar la actividad a los alumnos indicando las competencias abordadas, objetivos, metodología, evaluación y temporalización.

» Impartir clases teóricas y talleres de formación sobre las metodologías de trabajo y estrategias a emplear, así como las técnicas de investigación necesarias.

» Orientar, apoyar y seguir a los alumnos en los ejercicios, mediante consultas atendidas en clase y en tutorías.

» Determinar el nombramiento de dos coordinadores de la actividad.

» Evaluar la implementación de la calidad educativa y resultados obtenidos.

» Presentar los resultados de las evaluaciones. Celebrar un encuentro entre profesores y estudiantes para comunicar los resultados e incentivar a posibles nuevos participantes en este tipo de actividades de enseñanza-aprendizaje.

» Preparar una memoria final que comprenda todos los aspectos relevantes de esta experiencia educativa.

En todo momento, el profesorado tendrá en cuenta el clima de trabajo en el aula y potenciará el buen entendimiento, la colaboración, el respecto mutuo, la crítica constructiva o el intercambio de información, entre otras acciones, que aporten beneficios para la formación del alumnado (Toledo, 2010: web). En definitiva, cuidará el am- 
biente generado por la actividad docente, con el propósito de que la positividad haga más participes a los alumnos y que estos se encuentren cómodos y valorados adecuadamente (García y Manzano, 2010: 110).

Tareas del alumnado:

» Asistencia a la presentación de proyectos, clases teóricas y talleres de formación.

» Asistencia a tutorías (presenciales o virtuales).

» Distribución de grupos de trabajo y tareas.

» Elección de portavoz de grupo y distribución de tareas entre sus miembros.

» Búsqueda de fuentes de información y análisis de la misma.

» Desarrollo del proyecto adecuadamente con sus diferentes apartados.

» Exposición y defensa del trabajo.

» Auto-evaluación y evaluación entre iguales. Evaluación de la experiencia educativa y la metodología usada.

\subsection{Instrumentos de evaluación}

La evaluación se centra en el seguimiento de los trabajos de los alumnos, la elaboración de los mismos, el reparto de tareas, la responsabilidad, el compromiso adquirido, la colaboración y el resultado final. Se lleva a cabo empleando las siguientes técnicas e instrumentos:

» Calificación de la elaboración de trabajos y proyectos.

» Informes de las sesiones de trabajo.

» Sistemas de auto-evaluación (escrita e individual).

» Técnicas de observación (diarios, registros, etc.).

» Escalas de actitudes (para recoger opiniones, valores y habilidades sociales).

Los trabajos realizados se evalúan teniendo en cuenta principalmente los siguientes criterios: originalidad, fundamentación teórica, cumplimiento de las normas explicitas de la metodología activa propuesta, construcción del propio conocimiento, aportaciones hechas al área conocimiento, relación con la práctica profesional, claridad expositiva, bibliografía actualizada y amplia, referencias bibliográficas según normativa, estructuras gramaticales bien construidas y ortografia correcta.

La calificación final se ajusta al sistema general de evaluación que figura en la Guía docente de la signatura y su distribución en porcentajes (Universidad de Granada, $2012 \mathrm{web})$ :

" "Evaluación del grado de adquisición de competencias relacionadas con los contenidos de la materia a través de la evaluación continuada de las prácticas de creación, y trabajos propuestos mediante el seguimiento en el aula taller. 50 a $60 \%$.

» Resultado del estudio, lecturas, redacción y exposición de trabajos, cuadernos de campo, visitas de exposiciones, museos y ferias de arte 30 a $40 \%$.

» Asistencia y participación activa en las clases, seminarios y tutorías académicas. Hasta el 10\%".

Los instrumentos para la evaluación y seguimiento de la actividad son los siguientes: 
» Realización de cuestionarios a los alumnos sobre la calidad y adecuación de las actividades.

» Evaluación del profesor de la metodología de la enseñanza-aprendizaje, mediante la valoración de las calificaciones y las mejoras de las competencias adquiridas por los alumnos.

» Encuestas de evaluación continua de la actuación del docente, con la realización de varios cuestionarios anónimos hechos a los alumnos en diversos momentos de la docencia.

\section{Resultados}

Los resultados de esta experiencia docente, desarrollada durante el curso académico 2011-2012, analizados desde la valoración de las calificaciones obtenidas y la superación global de la asignatura, ofrecen una buena calificación cuantitativa de la misma (Tab. 1 y Graf. 1 A). Se han obtenido un $5 \%$ de Matriculas de Honor, que es el máximo permitido para un grupo docente de este número de matriculados, un 32,5\% de Sobresalientes e igual número de Notables, un 18,5\% de Aprobados, un $0 \%$ de Suspensos y han sido un $12,5 \%$ los alumnos No Presentados.

\begin{tabular}{|l|l|}
\hline \multicolumn{2}{|c|}{ Resultados por calificaciones del año 2011-12 } \\
\hline Matrícula de Honor & $2(5 \%)$ \\
\hline Sobresaliente & $13(32,5 \%)$ \\
\hline Notable & $13(32,5 \%)$ \\
\hline Aprobado & $7(18,5 \%)$ \\
\hline Suspenso & $0(0 \%)$ \\
\hline No presentado & $5(12,5 \%)$ \\
\hline Total $\mathbf{n}^{\mathbf{0}}$ alumnos & $\mathbf{4 0}$ \\
\hline
\end{tabular}

Tabla 1: Resultados y porcentajes de las calificaciones de la asignatura. Elaboración propia

Graf. 1A: Gráfico de las estadísticas en porcentajes de los resultados de las calificaciones de la asignatura (curso 2011-2012).

Graf. 1B: Gráfico de las estadísticas en porcentajes de los resultados de superación global de la asignatura (curso 2011-2012).

Elaboración propia.

Los resultado globales de superación de la asignatura (Tab. 2) muestran que han sido un $87,5 \%$ los alumnos que la han superado y un $12,5 \%$ los que no lo han hecho, debido a constar como No Presentados, por su falta de asistencia $\mathrm{o} / \mathrm{y}$ evaluación. Con respecto al curso académico anterior (2010-2011), ha au-

\section{Calificaciones del año 2011-12}

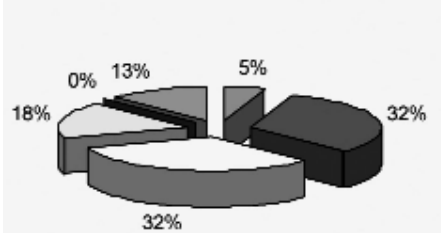

口 Matricula de Honor

- Sobresaliente

口 Notable

口Aprobado

- Suspenso

$\square$ No presentado

\section{Estadisticas por resultados}

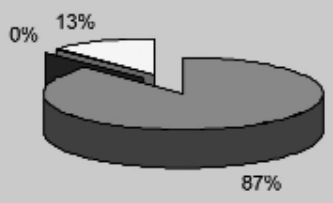

$\square$ Superados

$\square$ No Superados

$\checkmark$ No presentados 
mentado el índice de alumnos que han superado la asignatura en un 9,06\%, habiendo pasado del 78,44\% al 87,5\% y los Suspensos han pasado del 5,8\% al 0\%, siendo eliminados de la tabla. También se ha reducido la estadística de No Presentados en un $3,20 \%$ al cambiar del $15,7 \%$ al $12,5 \%$. En definitiva, el tanto por ciento de alumnos que han conseguido los objetivos y adquirido las capacidades y competencias requeridas en este nivel educativo ( $2^{\circ}$ curso) es más alto que el curso anterior (Graf. 1B) en proporción al número de matriculados.

\begin{tabular}{|l|l|l|}
\hline \multicolumn{1}{|c|}{ Resultados de superación } & \multicolumn{1}{|c|}{$\mathbf{2 0 1 1 - 1 2}$} & $\mathbf{2 0 1 0 - 1 1}$ \\
\hline Superados & $35(87,5 \%)$ & $40(78,44 \%)$ \\
\hline No superados & $0(0 \%)$ & $3(5,8 \%)$ \\
\hline No presentados & $5(12,5 \%)$ & $8(15,70 \%)$ \\
\hline Total $\mathbf{n}^{\mathbf{0}}$ alumnos & $\mathbf{4 0}$ & $\mathbf{5 1}$ \\
\hline
\end{tabular}

Tabla 2: Resultados globales y porcentajes de superación de la asignatura (curso 2011-2012 y 2010-2011. Elaboración propia.

En cuanto a la valoración de la actividad docente, el profesorado evalúa como positiva la adaptación de esta metodología a la mejora del aprovechamiento académico por la mayoría de los alumnos (Graf. 2), siendo 19 estudiantes los que lo han hecho muy bien, 13 bien, 3 mal y 5 muy mal.
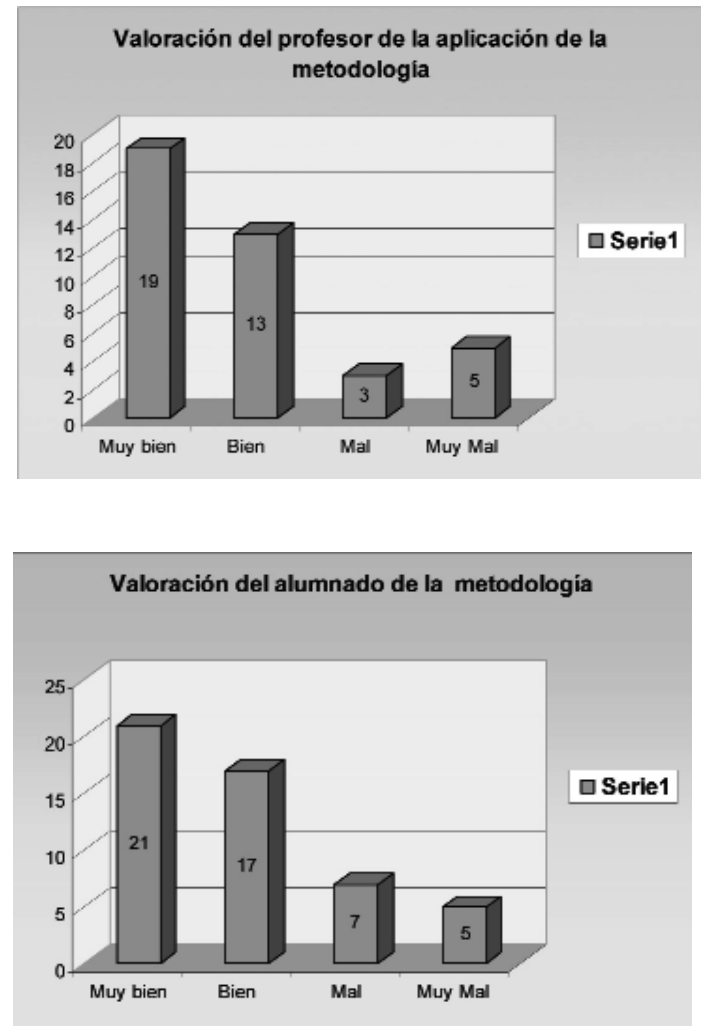

Graf. 2: Gráfico de valoración del profesorado de la adaptación de los alumnos a la metodología activa utilizada y la repercusión de ésta en su rendimiento académico. Eje vertical: $n^{\circ}$ de alumnos. Eje horizontal: valoración. Elaboración propia.

También el alumnado valora la metodología educativa utilizada como muy positiva (Graf. 3), con 21 estudiantes que la consideran muy buena, 17 buena, 7 mala y 5 muy mala.

Graf. 3: Gráfico de valoración del alumnado de la adaptación, utilidad y eficiencia de la metodología activa aplicada. Eje vertical: $\mathrm{n}^{\circ}$ de alumnos. Eje horizontal: valoración. Elaboración propia. 
Las calificaciones finales de la asignatura se han obtenido mediante la aplicación de técnicas de evaluación formativas continuas (inicial, media y final), cuantitativa basada en los resultados y cualitativa de las competencias desarrolladas por los alumnos.

\section{Conclusiones}

Durante el curso académico 2011-2012, se ha obtenido una mejora considerable en la calidad educativa de la asignatura Escultura III: Metodologías escultóricas (Grupo D), perteneciente al Grado en Bellas Artes de la Universidad de Granada, pues se han mejorado sus resultados académicos respecto al año anterior.

Se ha conseguido iniciar al alumno en la práctica de metodologías activas para el aprendizaje de forma individual y colaborativa grupal, que le benefician en su formación universitaria e inclusión laboral.

La metodología activa utilizada ha ayudado a mejorar la participación del alumnado en las actividades docentes y en su inclusión en el grupo de clase, lo que ha repercutido positivamente en el rendimiento global de la asignatura e individual del estudiante.

Se ha mejorado la adquisición de las competencias transversales que deben adquirir los alumnos a través de esta signatura, como la adaptación al trabajo en grupo, la responsabilidad compartida, la búsqueda y selección de información, la expresión verbal en público, la redacción escrita, la presentación de trabajos (oral y escrita), la autocrítica y la crítica grupal constructiva.

La titulación del Grado En Bellas Artes se ha visto beneficiada debido a la mejora de los rendimientos académicos y la mejora en la formación del alumnado.

La experiencia ha sido positiva y se recomienda sea puesta en práctica por otros grupos docentes.

\section{Referencias bibliográficas}

BENITO, Águeda y CRUZ, Ana (2005): Nuevas claves para la docencia universitaria en el Espacio Europeo de Educación Superior. Madrid, Narcea.

CEDEÑO, Blanca (2009): El papel del docente en el estímulo al proceso metacognitivo del estudiante en la resolución de problemas. Buenos Aires (Argentina), El Cid Editor apuntes, en: http://biblioteca.ugr.es/ [fecha de consulta 21 de octubre de 2012].

CONTRERAS, Luís Carlos; RODRÍGUEZ, José María y MORALES, Francisco José (eds. 2005): Innovamos juntos en la Universidad. Huelva, Servicio de Publicaciones de la Universidad de Huelva.

CROSS, Katheryn Patricia; MAJOR, Claire Howell y MANZANO, Pablo (coord., 2007): Técnicas de aprendizaje colaborativo. Madrid, Morata.

EXLEY, Kate y DENNICK, Reg (2009): Enseñanza en pequeños grupos en Educación Superior: tutorías, seminarios y otros agrupamientos. Madrid, Narcea.

GARCÍA, Begoña; MANZANO, María Encarnación (2010): Claves para aprender en un ambiente positivo y divertido: todo rueda mejor si se engrasa con humor. Madrid, Pirámide. 
GARCÍA-VALCÁRCEL, Ana (2001): Didáctica universitaria. Madrid, La Muralla. GÓMEZ, Miguel Ángel (2011): Enseñar en la universidad. Saberes, prácticas y textualidad. Bogotá, Ecoe Ediciones.

GROS, Begoña y ROMANÁ, Teresa (2004): Ser profesor. Palabras sobre la docencia universitaria. Barcelona, Octaedro/ICE-UB.

HERNÁNDEZ MARTÍN, Azucena y OLMOS MIGUELÁÑEZ, Susana (eds., 2011): Metodologías de aprendizaje colaborativo a través de las tecnologías. Salamanca, Ediciones Universidad de Salamanca, pp. 118-126.

HERREID, Cyde Freeman. (1994): Case Studies in Science, a Novel Method of Science Education, en Journal of College Science Teaching, 23 (4), 221-229.

LÓPEZ NOGUERO, Fernando (2005): Metodología participativa en la enseñanza universitaria. Madrid, Narcea.

MAZARÍO TRIANA, Israel (2009): La resolución de problemas: un reto para la educación contemporánea. Ciudad de La Habana, Editorial Universitaria, en: http://biblioteca.ugr.es/ [fecha de consulta 21 de octubre de 2012].

PAGÈS, Teresa; CORNET, Albert y PARDO, Jordi (coords., 2010): Buenas prácticas docentes en la universidad: Modelos y experiencias en la Universidad de Barcelona. Barcelona, Octaedro-ICE.

RUÉ, Joan. (2007): Enseñar en la Universidad: el EEES como reto para la educación superior. Barcelona, Narcea.

TOLEDO MORALES, Purificación (2000). "El ambiente universitario. Estudio descriptivo y comparativo del clima del aula de la Universidad de Jaén" en Edición Digital@tres: http://gdus.us.es/proyectos20092010/710041_658_1729.pdf [fecha de consulta: 2 de septiembre de 2012].

UBIETO, María Isabel (coord.); DOMINGO, María; GARCÍA, Francisco Javier y PAULO, Antonio (2008): Practicar con la teoría: metodologías activas en información y documentación en el marco del Espacio Europeo de Educación Superior. Zaragoza, Prensas Universitarias de Zaragoza.

UNIVERSIDAD DE GRANADA (2010): Grado en Bellas Artes, Universidad de Granada. Granada, en: http://vicengp.ugr.es/pages/_grados-verificados/04bellasartesverificado [fecha de consulta 23 de octubre de 2012].

UNIVERSIDAD DE GRANADA (2012): Guia Docente de la asignatura Escultura III: Metodologías escultóricas, en: http://grados.ugr.es/bellasartes/pages/infoacademica/guiasdocentes/segundo-curso/0207guiadocenteesculturaiiimetodologiasescultoricas201112/ [fecha de consulta: 3 de septiembre de 2012].

UNIVERSIDAD REY JUAN CARLOS (2008): Calidad e innovación docente en el marco de la Convergencia Europea. Madrid: Consejo Social. Vicerrectorado del Profesorado, Titulaciones, Ordenación Académica, Coordinación y Campus, en: http://www.dykinson-on-line.com/calidad_e_innovacion_docente_en_el_marco_de _la_convergencia_europea.ebook5961 [fecha de consulta: 22 de octubre de 2012]. 
VILLAR, Luis Miguel (coord., 2004): Programa para la mejora de la docencia universitaria. Madrid, Pearson Prentice Hall.

ZABALZA, Miguel Ángel (2007): La enseñanza universitaria. El escenario y sus protagonistas. Madrid, Nancea.

ZABALZA BEREZA, Miguel Ángel y ZABALZA CERDEIRIÑA, María (2010): Planificación de la docencia en la Universidad: elaboración de las guías docentes de las materias. Madrid, Narcea.

\section{María del Carmen BELLIDO MÁRQUEZ}

Universidad de Granada

cbellido@ugr.es

Departamento de Escultura

Profesora Ayudante a Doctor 